\title{
ORIGINAL RESEARCH \\ Human Subinsular Asymmetry Studied by Diffusion Tensor Imaging and Fiber Tracking
}

S. Rodrigo
O. Naggara

C. Oppenheim

N. Golestani

C. Poupon

Y. Cointepas

J.F. Mangin

D. Le Bihan

J.F. Meder
BACKGROUND AND PURPOSE: Our aim was to improve our understanding of the subinsular white matter microstructural asymmetries in healthy right-handed subjects. Structural brain asymmetries could be related to functional asymmetries such as hemisphere language dominance or handedness. Besides the known gray matter asymmetries, white matter asymmetries could also play a key role in the understanding of hemispheric specialization, notably that of language.

MATERIALS AND METHODS: White matter asymmetries were studied by diffusion tensor imaging at 1.5T (41 diffusion-gradient directions; b-value set to $700 \mathrm{~s} / \mathrm{mm}^{2}$; matrix, 1282; in-plane resolution, $1.875 \times 1.875 \mathrm{~mm}$; section thickness, $2.0 \mathrm{~mm}$ ) and fiber tracking (BrainVISA software). The main white matter bundles passing through the subinsular area were segmented, and fractional anisotropy (FA) was measured along each of the segmented bundles.

RESULTS: In line with published results, we found an asymmetry of the arcuate fasciculus and the subinsular white matter, namely left-greater-than-right FA in right-handed controls. Furthermore, by segmenting major tracts coursing through this region, we showed that the subinsular portions of the uncinate fasciculus (UF) and the inferior occipitofrontal fasciculus (IOF) contribute to this FA asymmetry. Those tracts have been reported to be likely implicated in the language network.

CONCLUSION: Because the left hemisphere hosts language functions in most right-handers, the significant leftward asymmetry observed within the arcuate fasciculus, the subinsular part of the UF and IOF may be related to the hemispheric specialization for language.
S tructural brain asymmetries in the size of regions of the cerebral cortex or in brain white matter could be related to functional asymmetries such as handedness or hemispheric language dominance. There are structural asymmetries in regions of the language network, including the primary auditory cortex, the planum temporale, Wernicke area, and the angular gyrus. ${ }^{1-5}$ These cortical asymmetries, mainly reflecting larger volumes in the left hemisphere, suggest a link between cortical size/shape and hemispheric functional lateralization. Moreover, it has been shown that white matter differences drive some of the asymmetries in regions of the auditory cortex and of the language network, ${ }^{6}$ including the Heschl gyrus ${ }^{4,7}$ and the planum temporale. ${ }^{8}$ White matter structure could, therefore, play a key role for the understanding of hemispheric specialization, notably that of language. In addition, larger white matter volumes in the left hemisphere could provide a structural basis for greater intrahemispheric connectivity. ${ }^{9}$

Diffusion tensor imaging (DTI) is an MR imaging technique measuring random displacements of water molecules. ${ }^{10,11}$ Anisotropy measures, such as fractional anisotropy (FA) provided by DTI, could reflect organization of white matter fiber bundles. ${ }^{12}$ DTI studies have revealed an FA asymmetry of the arcuate fasciculus, ${ }^{13,14}$ a white matter tract connecting the anterior and posterior language poles. These asymmetries could consequently be related to hemispheric

Received September 7, 2006; accepted after revision February 5, 2007.

From the Département d'Imagerie Morphologique et Fonctionnelle (S.R., O.N., C.O., J.F. Meder), Faculté de Médecine, Université Paris-Descartes, Centre Hospitalier Sainte-Anne, Paris, France; and Unité de Neuro-Imagerie Anatomo-Fonctionnelle (S.R., C.O., N.G., C.P., Y.C., J.F. Mangin, D.L.B.) and INSERM U 562, Cognitive Neuroimaging, (C.O., N.G.), Service Hospitalier Frederic Joliot, CEA, Orsay, France.

Please address correspondence to Catherine Oppenheim, MD, PhD, Département d'Imagerie Morphologique et Fonctionnelle, Centre Hospitalier Sainte-Anne, 1 rue Cabanis, 75674 Paris cedex 14, France; e-mail: c.oppenheim@ch-sainte-anne.fr

DOI 10.3174/ajnr.A0584 specialization for speech. ${ }^{15}$ Recently, a study combining DTI and functional MR imaging (fMRI) reported that righthanded subjects had a leftward asymmetry of the arcuate fasciculus, which correlated with left-lateralized fMRI activation using language tasks. ${ }^{16}$

As recently reviewed by Catani and ffytche, ${ }^{17}$ the existence of disconnection syndromes such as conduction aphasia underlines the importance of investigating brain function as a distributed network and also the importance of trying to better understand white matter pathways. Apart from work on the arcuate fasciculus, the study of subinsular white matter fibers and their cortical connections and functional roles has been a focus of interest. Indeed, disruption of the cortical communications could explain the deficits in the processing of auditory and visual stimuli in developmental dyslexia. ${ }^{18}$ Similarly, infarction of the insular cortex ${ }^{19,20}$ and lesions of the extreme capsule are proposed explanations for conduction aphasia, ${ }^{21}$ which is regarded as a disruption of communication between anterior and posterior cortical language regions. In support of this, Cao et $\mathrm{al}^{22}$ examined the subinsular region and found left-greater-than-right anisotropy in right-handed healthy individuals. As stated by these authors, these microstructural subinsular left-right asymmetries could be related to left hemispheric lateralization for speech in language in right-handed individuals. In their study, anisotropy indices were measured in manually drawn regions of interest (ROI). ${ }^{22}$ Their approach allows examining the global microstructure of the subinsular area. It does not, however, identify pathways that course through the region-of-interest boundaries and contribute to microstructural asymmetries.

DTI provides a main eigenvector, which can be regarded as the main fiber-orientation estimate within a voxel. Fiber tractography algorithms connect the estimated fiber orientation between voxels, thereby allowing inferences to be made about the course of fiber bundles. ${ }^{23-26}$ Our aim in this study was to 
improve our understanding of the subinsular white matter microstructural asymmetries in healthy right-handed subjects. We did this by tracking the main white matter bundle passing through this subinsular area and measuring FA along each of the segmented bundles. We additionally checked the leftward asymmetry found for the arcuate fasciculus. ${ }^{16}$

\section{Methods}

Eighteen right-handed healthy subjects, 5 men and 13 women, were studied (mean age, $24 \pm 3.3$ years). Handedness was assessed by using the Edinburgh inventory, ${ }^{27}$ (mean $\pm \mathrm{SD}$ : $93.7 \pm 7.1$ ). All subjects gave written informed consent to participate in the study, which was approved by the local ethics committee. Brain data were acquired by using a 1.5T MR imaging scanner (Signa Excite 1.5T; GE Healthcare, Milwaukee, Wis). The diffusion-weighted sequence parameters were as follows : spin-echo along with echo-planar imaging (EPI); axial contiguous sections covering the whole brain (bicommissural plane); 41 diffusion-gradient directions; b-value set to $700 \mathrm{~s} / \mathrm{mm}^{2}$; matrix, $128^{2}$; in-plane resolution, $1.875 \times 1.875 \mathrm{~mm}$; section thickness, 2.0 mm; TE, $66.6 \mathrm{~ms}$; TR, $2000 \mathrm{~ms}$; single shot; FOV, $24 \mathrm{~cm}$. Diffusionweighted data were analyzed by using BrainVISA/Anatomist software (http://brainvisa.info). ${ }^{28}$ After correction of the spatial distortions induced by eddy currents, a robust tensor estimation was computed. ${ }^{11,29} \mathrm{FA}$, mean diffusivity (MD), and direction color-coded maps were then computed. ${ }^{11,30}$

\section{Subinsular 3D Region of Interest}

The first step consisted of drawing 3D ROIs within the subinsular white matter bilaterally on the T2-weighted image from diffusion data $\left(\mathrm{b}=0 \mathrm{~s} / \mathrm{mm}^{2}\right)$. The $3 \mathrm{D} \mathrm{ROI}_{\text {subinsular }}$ was segmented by using previously described criteria. ${ }^{22}$ This region lies between the insular cortex and the putamen and includes the external capsule, the extreme capsule, and the claustrum, which is a layer of gray matter that lies between these 2 capsules. Only axial sections containing both the insular cortex and the putamen landmarks were used. The volume, FA, and MD values were then extracted bilaterally from the $3 \mathrm{D} \mathrm{ROI}_{\text {subinsular }}$. As discussed by Cao et al, ${ }^{22}$ asymmetries in the thickness of these gray matter structures and partial volume averaging from gray matter, the putamen, and the insular cortex could influence subinsular asymmetries. We, therefore, also checked our results by masking gray matter pixels. The mask was computed by thresholding FA maps and keeping only pixels with FA values $\geq 0.35$. This value was selected by gradually increasing the FA threshold, starting from $0.15 .^{31}$ The threshold was then set to 0.35 because on the basis of anatomic landmarks, ${ }^{32}$ no gray matter pixels remained.

The second step consisted of identifying the main white matter bundles passing through the $3 \mathrm{D} \mathrm{ROI}_{\text {subinsular }}$ and then calculating the mean FA and MD values along these bundles. To do so, we used the

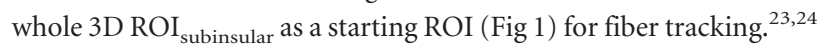
Fiber tracking relied on the fiber assignment by continuous-tracking algorithm (Mori et al, $1999^{24}$ ), by using an angular threshold of $<45^{\circ}$ (keeping only voxels with $\mathrm{FA}>0.2$ to restrict the fibers assigned to white matter) and 32 seed points per ROI. The tracking delineated 2 main bundles.

\section{Uncinate Fasciculus}

One white matter pathway coursed lateral to the amygdala and curved within the subinsular white matter to the frontobasal region. This tract followed the known anatomic pathway of the uncinate fasciculus $(\mathrm{UF})^{32,33}$ and was thus named after this bundle (Fig 2). To isolate the

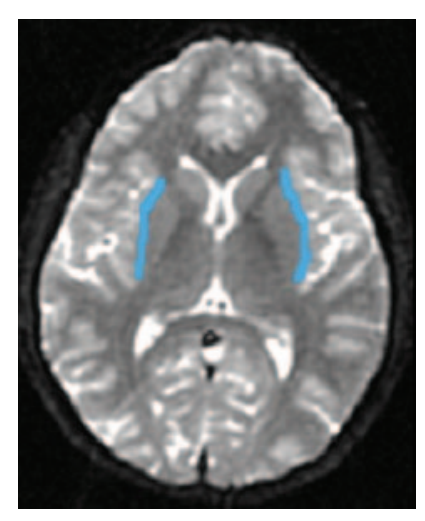

Fig 1. Subinsular 3D region of interest (b/ue) drawn on an axial T2-weighted echo-planar image.

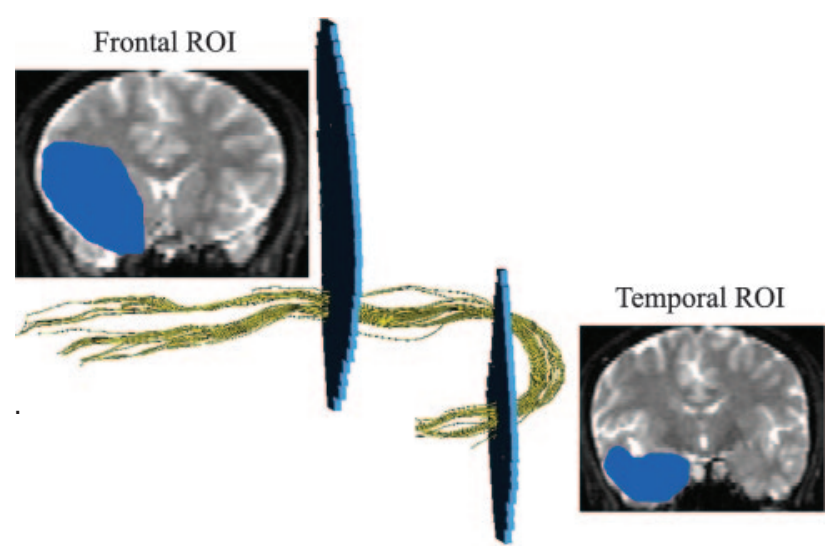

Fig 2. Lateral view of the UF DTI-based tracking (yellow), constrained by frontal and temporal regions of interest (ROI, blue), drawn on coronal views.

UF from other bundles, the fiber tracking was constrained by the 2 following ROIs, which were manually drawn on each subject's colorcoded diffusion map: 1) a large temporal ROI drawn on a coronal section located just behind the amygdaloid body, and 2) a large frontal ROI drawn on a coronal section located half-way between the anterior margin of the insula and the genu of the corpus callosum (Fig 2). Only fibers passing through these 2 ROIs were then included.

\section{Inferior Occipitofrontal Fasciculus}

The other white matter pathway identified by the tracking coursed from the occipital pole through the $3 \mathrm{D} \mathrm{ROI}_{\text {subinsular }}$ just behind the UF and curved toward the frontolateral region. This tract followed the known anatomic pathway of the inferior occipitofrontal fasciculus $(\mathrm{IOF})^{32,33}$ and was thus named after this bundle (Fig 3). To isolate the IOF from other bundles, the fiber tracking was constrained by the 2 following ROIs, which were manually drawn on each subject's colorcoded diffusion maps: 1) a large occipital ROI drawn on a coronal section located just behind the splenium of the corpus callosum (Fig 3 ), and 2) the same frontal ROI as that used for the UF tractography. Only fibers passing through these 2 ROIs were then included.

\section{Arcuate Fasciculus}

For tracking the arcuate fasciculus, we used the 2 following ROIs, which were manually drawn on each subject's color-coded diffusion maps: 1) a large ROI drawn on a coronal section at the level of the parietal operculum, and 2) a ROI drawn on an axial section lateral to the ventricular trigone. Only fibers passing through these 2 ROIs were then included. 


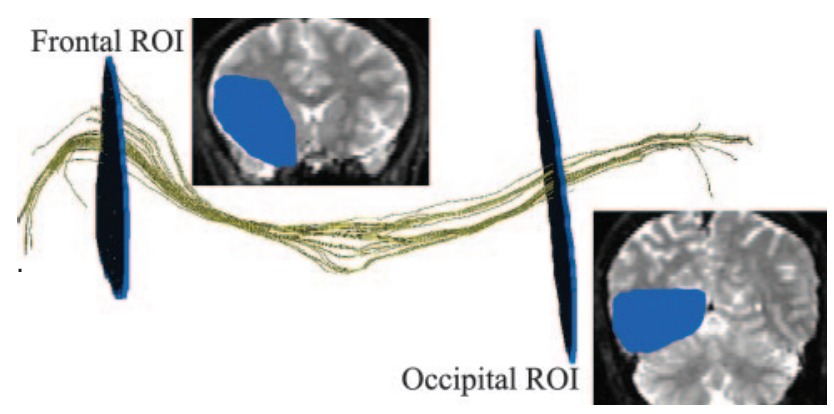

Fig 3. Lateral view of the IOF DTI-based tracking (yellow), constrained by frontal and occipital regions of interest (ROI, blue), drawn on coronal views.

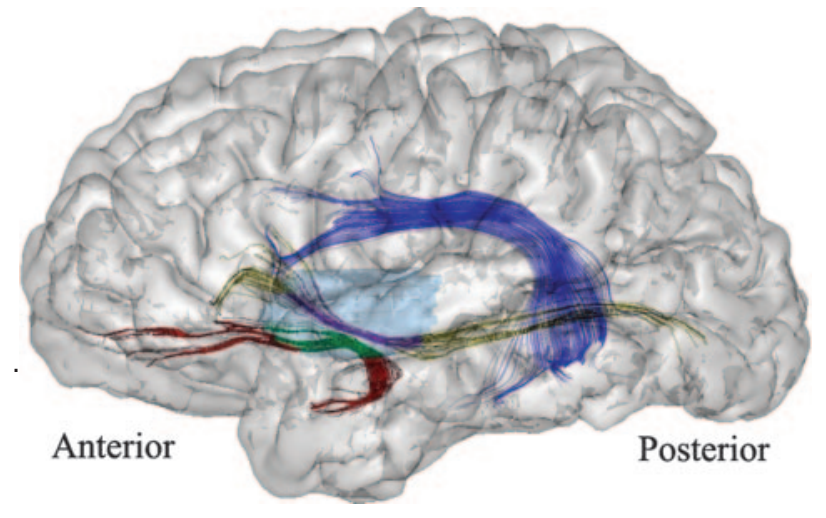

Fig 4. Illustration of the fiber tracking projected on a left brain hemisphere (lateral view). Arcuate fasciculus tracking is displayed in dark blue. Segmentation of the UF and IOF

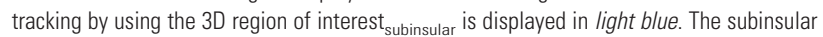
portions of the UF and IOF are displayed in green and purple, respectively. Their extrainsular portions of the UF and IOF are displayed in red and in yellow, respectively.

\section{FA and MD Measurements}

We first determined the mean FA and MD in the 3D ROI subinsular $_{\text {, as }}$ well as in the arcuate fasciculus, UF, and IOF, bilaterally. We then split the UF and IOF into 2 portions by using the $3 \mathrm{D} \mathrm{ROI}_{\text {subinsular }}$ as a mask. For each of these tracts, there was thus a subinsular segment and extrainsular segments (Fig 4). Mean FA and MD were calculated along these UF and IOF subregions.

\section{Statistical Analyses}

The association between mean $\mathrm{FA}$ or $\mathrm{MD}$ values in the $3 \mathrm{D}$

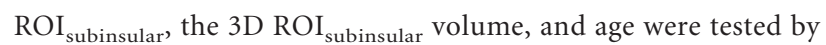
using Pearson product moment correlations in the right and left hemispheres separately. To test for FA asymmetries, we compared group right and left FA and MD values from the 3D ROIs subinsular; from the whole arcuate fasciculus, UF, and IOF; and from the UF and IOF subregions by using paired Student $t$ tests.

\section{Results}

\section{D Region of Interest subinsular $_{\text {. }}$}

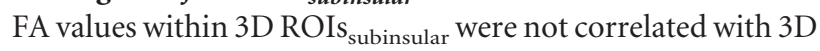

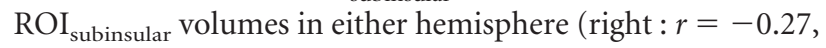
$P=.26$; left $: r=-0.035, P=.44$ ), or with subjects' age (right $: r=-0.17, P=.49$; left $: r=-0.39, P=.11)$. We found a significant difference $(P=.007)$ between left and right FA. The left $>$ right FA asymmetry remained significant after gray matter masking $(P<.001)$. The difference between left and right $\mathrm{MD}$ values was not significant, either before or after gray matter masking.

\section{UF and IOF}

For the whole UF (Table), FA was greater in the right compared with the left hemisphere though not significantly different $(P=.06)$; this pattern was opposite of that found for the 3D $\mathrm{ROI}_{\text {subinsular. }}$ For the whole IOF, FA was significantly greater in the left compared with the right hemisphere $(P<$ $.001)$, like that found for the $3 \mathrm{D} \mathrm{ROI}_{\text {subinsular. }}$ As indicated in the Table, side-to-side MD values were significantly different for the whole UF $(P=.01)$, but not for the IOF.

\section{Subinsular and Extrainsular UF and IOF}

For the subinsular parts of the UF and IOF, we found significantly left-greater-than-right FA asymmetry. Conversely, we found a significantly right-greater-than-left FA in the extrainsular part of the UF and no significant FA asymmetry for the IOF extrainsular part. Side-to-side MD values were significantly different for the extrainsular UF $(P=.04)$ but not for the other split bundles (Table).

\section{Arcuate Fasciculus}

For the arcuate fasciculus, we found a significant left-greaterthan-right FA $(P<.001)$ without corresponding significant MD asymmetry.

\section{Discussion}

Our results first confirm the left-greater-than-right FA of the arcuate fasciculus in right-handed healthy participants, previously reported. ${ }^{16}$ In line with the results of Cao et al, ${ }^{22}$ we found an asymmetry of the subinsular white matter, namely a left-greater-than-right FA asymmetry in right-handed healthy participants. As suggested by these authors, there may be an association between this left-right asymmetry of the subinsular area and the left hemispheric lateralization of language and speech in right-handed individuals. Furthermore, by segmenting 2 major white matter tracts coursing through this region, we showed that the subinsular portions of the UF and IOF both contribute to this FA asymmetry. Because these pathways may be part of the language network, these results may contribute to our understanding of relationships between brain white matter microstructure and brain function. Before commenting on such relationships, we will first discuss our findings for each of the segmented bundles.

\section{UF}

Although a left-greater-than-right FA was found within the subinsular segments of the UF and of the IOF, surprisingly, we found a reversed pattern of FA asymmetry (ie, right $>$ left) when the UF was considered in its entire length or when only its extrainsular portion was examined. By showing that the direction of FA asymmetry varies along the UF, our findings might reconcile some apparently conflicting results in the literature. First, a postmortem study has shown that the right UF is larger and contains more fibers than the left UF, suggesting a greater right frontotemporal connectivity in healthy adult human brains. ${ }^{34}$ In line with this, voxel-based analysis of DTI data has revealed a right-greater-than-left FA at the level of the UF stem and its inferior parts. ${ }^{35}$ These asymmetries, however, may be influenced by the effect of the size of the spatial smoothing kernel, ${ }^{35,36}$ a value determined during 1 of the steps of voxel-based analyses. Indeed, left-greater-than-right FA 


\begin{tabular}{|c|c|c|c|c|c|c|}
\hline \multicolumn{7}{|c|}{ DTI measurements along the UF, IOF, and arcuate fasciculi } \\
\hline & \multicolumn{2}{|c|}{ FA } & \multirow[b]{3}{*}{$P$} & \multicolumn{2}{|c|}{$M D^{*}$} & \multirow[b]{3}{*}{$P$} \\
\hline & \multicolumn{2}{|c|}{ Mean \pm SD } & & \multicolumn{2}{|c|}{ Mean \pm SD } & \\
\hline & Left & Right & & Left & Right & \\
\hline $\begin{array}{l}\text { 3D ROI subinsular } \\
\text { UF }\end{array}$ & $0.34 \pm 0.06$ & $0.30 \pm 0.04$ & .007 & $8.76 \pm 0.30$ & $8.76 \pm 0.61$ & N.S. \\
\hline Whole & $0.44 \pm 0.01$ & $0.45 \pm 0.02$ & .06 & $9.01 \pm 0.20$ & $8.84 \pm 0.16$ & .01 \\
\hline Subinsular & $0.50 \pm 0.06$ & $0.44 \pm 0.06$ & .006 & $8.85 \pm 0.68$ & $8.90 \pm 0.50$ & N.S. \\
\hline Extrainsular & $0.44 \pm 0.03$ & $0.46 \pm 0.02$ & .017 & $9.10 \pm 0.20$ & $8.91 \pm 0.28$ & .04 \\
\hline IOF & & & & & & \\
\hline Whole & $0.56 \pm 0.02$ & $0.53 \pm 0.02$ & $<.001$ & $8.96 \pm 0.24$ & $8.82 \pm 0.21$ & N.S. \\
\hline Subinsular & $0.54 \pm 0.04$ & $0.51 \pm 0.02$ & .02 & $8.46 \pm 0.21$ & $8.38 \pm 0.24$ & N.S. \\
\hline Extrainsular & $0.55 \pm 0.08$ & $0.56 \pm 0.06$ & N.S. & $8.89 \pm 0.86$ & $8.92 \pm 0.25$ & N.S. \\
\hline Arcuate fasciculus & $0.53 \pm 0.03$ & $0.48 \pm 0.04$ & $<.001$ & $8.84 \pm 0.30$ & $8.48 \pm 0.61$ & N.S. \\
\hline
\end{tabular}

Note:-ROI indicates region of interest; UF, uncinate fasciculus; IOF, inferior occipito fasciculus; N.S., nonsignificant.

${ }^{*} \mathrm{MD}$ is $10^{-10} \mathrm{~m}^{2} / \mathrm{s}$.

was observed in the superior portion of the UF by the same group when reducing the size of the smoothing kernel. ${ }^{35}$ This latter result is consistent with our findings and with those of Kubicki et $\mathrm{al},{ }^{37}$ who, by using a ROI-based analysis, found a left-greater-than-right anisotropy of the subinsular UF. Discrepancies between DTI results may also be explained by variations of diffusion parameters along the course of a given white matter tract, as previously observed for the cingulum. ${ }^{38}$ Anatomically, the UF is highly packed within its subinsular portion, which should lead to high anisotropy. Conversely, in its extrainsular portion, the UF crosses other pathways, such as fibers arising from the anterior corpus callosum and from the arcuate fasciculus in the temporal region. ${ }^{32}$ Such fiber crossings should lead to lower anisotropy values. These spatial variations in white matter microstructure may account for the different asymmetry patterns that we observed for the extrainsular-versus-the subinsular portions of the UF.

\section{IOF}

DTI-based tracking of the IOF is feasible, as previously shown..$^{39}$ As expected, we showed left-greater-than-right FA in the whole IOF and in its subinsular part. This asymmetry was no longer significant when considering only the extrainsular part. To our knowledge, no previous studies have reported FA values along the length of the IOF in a healthy population; however, FA values at the level of the temporal stem of the right IOF have been reported. ${ }^{40}$ These were slightly lower than ours, and the discrepancy could be due to differences in the pulse sequence, section thickness, image analysis, or to the region/subregions examined along the course of IOF.

\section{Structure Function Relationship}

Interhemispheric white matter asymmetries may be an important anatomic basis for hemispheric specialization. Axonal white matter fibers connecting cortical regions involved in speech, language, and reading may demonstrate asymmetry in their microstructural organization, the degree of axon myelination, or the integrity of axonal cell membranes. ${ }^{22}$ The use of DTI and of fiber tracking has allowed us to study white matter tracts that might be damaged in disconnection syndromes such as conduction aphasia. ${ }^{21}$ Cao et al ${ }^{22}$ previously examined insular white matter, a region relevant for the language network. As expected, they showed a leftward asymmetry of in- sular white matter in right-handed healthy volunteers. ${ }^{22}$ Within this area, we further identified 2 main bundles, the UF and IOF, also potentially implicated in the language network. From an anatomic point of view, the UF connects the orbitofrontal cortex to the medial part of the temporal pole and the dorsolateral frontal cortex to the first and second temporal gyri. ${ }^{32}$ Its functional role in the language network remains unclear. In surgery of intractable seizures, anterior temporal lobectomy involves the resection of the temporal pole and part of the UF. It has been shown that category-specific naming deficits following a left temporal lobectomy were more pronounced than those after a right temporal resection. ${ }^{41}$ This finding suggests a functional difference or asymmetry between left and right frontotemporal connectivity, likely involving the UF. ${ }^{41}$ The left-right asymmetry that we observed in the UF is consistent with the greater deficit observed after left temporal lobectomy. The UF has also been the focus of interest in schizophrenia. In this population, functional brain imaging studies using verbal tasks support a frontotemporal disconnection. ${ }^{42-44}$ Absence of left-right DTI asymmetry along the UF in schizophrenia compared with that in controls ${ }^{37}$ further supports the frontotemporal disconnection hypothesis.

On the basis of modern primate electrophysiologic and tracer injection studies, ${ }^{45}$ Parker et al ${ }^{14}$ used DTI-based fiber tracking to delineate 2 parallel auditory language pathways: a dorsal-arcuate fasciculus and a ventral fasciculus, coursing through the external capsule. The dorsal-arcuate fasciculus is considered as a main pathway within the perisylvian language network, as was recently reviewed. ${ }^{17,46}$ Interestingly, a structure-function relationship is supported by the correlation between the leftward structural lateralization of the arcuate fasciculus using DTI and the leftward language lateralization in both frontal and temporal lobes using fMRI. ${ }^{16}$

The other pathway, the ventral one, which connects the Wernicke and Broca areas, likely corresponds to the IOF. ${ }^{14}$ In line with this, corticosubcortical electrostimulations support the idea that a main ventral subcortical pathway in the dominant hemisphere is important for the semantic processing system. ${ }^{47}$ The course of this pathway, which connects the posterosuperior temporal region with the orbitofrontal and dorsolateral prefontal regions, might anatomically correspond, in part, to the inferior fronto-occipital fasciculus. ${ }^{47}$ 


\section{Limitations}

In this article, we assumed that the 2 segmented pathways corresponded to the UF and the IOF. This assumption was based on the similarity between the course of these pathways and previous anatomic descriptions. However, due to DTI limitations in properly modeling crossing fibers, we cannot exclude the possibility that some of the segmented pixels might belong to other pathways. ${ }^{10,11}$ Alternatively, due to partial volume effects at the boundaries, the segmented pathways may not include all of the UF or IOF. These limitations might have biased our FA measurements. They may also explain the fact that we only identified 2 bundles within the subinsular area. We did not identify smaller pathways, such as shortassociation fibers arising from the UF or the IOF or fibers arising from the arcuate fasciculus that anatomically course through the subinsular area. ${ }^{32}$ This shortcoming may be due to the ROIs used to select the tracks or to the tensorial model used in this work. The use of more advanced diffusion models, such as Q-ball imaging, ${ }^{48}$ could determine if these small pathways participate in the left-right asymmetry of the subinsular area.

\section{Conclusions}

We confirmed the left-greater-than-right FA of the arcuate fasciculus in right-handers; and within the subinsular area, we identified 2 pathways, the UF and IOF, likely implicated in the language network. We showed that FA measurements along the UF and IOF can at least in part explain the subinsular left-greater-than-right FA asymmetry in right-handers. In this population, these bundles are likely more coherently organized in the left hemisphere. Although the relationship between manual preference and language lateralization is a stilldebated issue, it is known that the left hemisphere hosts language functions in most right-handers. ${ }^{49}$ Consequently, the significant leftward asymmetry observed in the arcuate fasciculus, UF, and IOF at the subinsular level may be related to hemispheric specialization for language. Our results highlight the importance of considering not only the arcuate fasciculus but also the UF and IOF when studying structurefunction relationships. Future studies combining fMRI and DTI will help to clarify the association between brain functional lateralization and white matter asymmetries.

\section{References}

1. Galaburda AM, Sanides F, Geschwind N. Human brain: cytoarchitectonic leftright asymmetries in the temporal speech region. Arch Neurol 1978;35:812-17

2. Galaburda AM, LeMay M, Kemper TL, et al. Right-left asymmetrics in the brain. Science 1978;199:852-56

3. Geschwind N, Levitsky W. Human brain: left-right asymmetries in temporal speech region. Science 1968;161:186-87

4. Penhune VB, Zatorre RJ, MacDonald JD, et al. Interhemispheric anatomical differences in human primary auditory cortex: probabilistic mapping and volume measurement from magnetic resonance scans. Cereb Cortex 1996;6:661-72

5. Watkins KE, Paus T, Lerch JP, et al. Structural asymmetries in the human brain: a voxel-based statistical analysis of 142 MRI scans. Cereb Cortex 2001;11:868-77

6. Pujol J, Lopez-Sala A, Deus J, et al. The lateral asymmetry of the human brain studied by volumetric magnetic resonance imaging. Neuroimage 2002; 17:670-79

7. Golestani N, Molko N, Dehaene S, et al. Brain structure predicts the learning of foreign speech sounds. Cereb Cortex 2007;17:575-82. Epub 2006 Apr 7

8. Anderson B, Southern BD, Powers RE. Anatomic asymmetries of the posterior superior temporal lobes: a postmortem study. Neuropsychiatry Neuropsychol Behav Neurol 1999;12:247-54
9. Herve PY, Crivello F, Perchey G, et al. Handedness and cerebral anatomical asymmetries in young adult males. Neuroimage 2006;29:1066-79

10. Le Bihan D, Mangin JF, Poupon C, et al. Diffusion tensor imaging: concepts and applications. J Magn Reson Imaging 2001;13:534-46

11. Pierpaoli C, Jezzard P, Basser PJ, et al. Diffusion tensor MR imaging of the human brain. Radiology 1996;201:637-48

12. Beaulieu C. The basis of anisotropic water diffusion in the nervous system: a technical review. NMR Biomed 2002;15:435-55

13. Buchel C, Raedler T, Sommer M, et al. White matter asymmetry in the human brain: a diffusion tensor MRI study. Cereb Cortex 2004;14:945-51

14. Parker GJ, Luzzi S, Alexander DC, et al. Lateralization of ventral and dorsal auditory-language pathways in the human brain. Neuroimage 2005;24:656-66

15. Matsumoto R, Nair DR, LaPresto E, et al. Functional connectivity in the human language system: a cortico-cortical evoked potential study. Brain 2004;127(pt 10):2316-30. Epub 2004 Jul 21

16. Powell HW, Parker GJ, Alexander DC, et al. Hemispheric asymmetries in language-related pathways: a combined functional MRI and tractography study. Neuroimage 2006;32:388-99

17. Catani M, ffytche DH. The rises and falls of disconnection syndromes. Brain 2005;128:2224-39. Epub 2005 Sep 1.

18. Klingberg T, Hedehus M, Temple E, et al. Microstructure of temporo-parietal white matter as a basis for reading ability: evidence from diffusion tensor magnetic resonance imaging. Neuron 2000;25:493-500

19. Metter EJ, Riege WH, Hanson WR, et al. Subcortical structures in aphasia: an analysis based on (F-18)-fluorodeoxyglucose, positron emission tomography, and computed tomography. Arch Neurol 1988;45:1229-34

20. Shuren J. Insula and aphasia. J Neurol 1993;240:216-18

21. Damasio H, Damasio AR. The anatomical basis of conduction aphasia. Brain 1980;103:337-50

22. Cao Y, Whalen S, Huang J, et al. Asymmetry of subinsular anisotropy by in vivo diffusion tensor imaging. Hum Brain Mapp 2003;20:82-90

23. Conturo TE, Lori NF, Cull TS, et al. Tracking neuronal fiber pathways in the living human brain. Proc Natl Acad Sci U S A 1999;96:10422-27

24. Mori S, Crain BJ, Chacko VP, et al. Three-dimensional tracking of axonal projections in the brain by magnetic resonance imaging. Ann Neurol 1999;45:265-69

25. Behrens TE, Johansen-Berg H, Woolrich MW, et al. Non-invasive mapping of connections between human thalamus and cortex using diffusion imaging. Nat Neurosci 2003;6:750-57

26. Poupon C, Clark CA, Frouin V, et al. Regularization of diffusion-based direction maps for the tracking of brain white matter fascicles. Neuroimage 2000;12:184-95

27. Oldfield RC. The assessment and analysis of handedness: the Edinburgh inventory. Neuropsychologia 1971;9:97-113

28. Cointepas Y, Mangin JF, Garnero L, et al. BrainVISA: software platform for visualization and analysis of multi-modality brain data. Neuroimage 2001;13:S98

29. Mangin JF, Poupon C, Clark C, et al. Distortion correction and robust tensor estimation for MR diffusion imaging. Med Image Anal 2002;6:191-98

30. Pajevic S, Pierpaoli C. Color schemes to represent the orientation of anisotropic tissues from diffusion tensor data: application to white matter fiber tract mapping in the human brain. Magn Reson Med 1999;42:526-40

31. Stieltjes B, Kaufmann WE, van Zijl PC, et al. Diffusion tensor imaging and axonal tracking in the human brainstem. Neuroimage 2001;14:723-35

32. Dejerine J. Anatomie des Centres Nerveux. Paris, France: Rueff et Cie; 1895

33. Kier EL, Staib LH, Davis LM, et al. MR imaging of the temporal stem: anatomic dissection tractography of the uncinate fasciculus, inferior occipitofrontal fasciculus, and Meyer's loop of the optic radiation. AJNR Am J Neuroradiol 2004;25:677-91

34. Highley JR, Walker MA, Esiri MM, et al. Asymmetry of the uncinate fasciculus: a post-mortem study of normal subjects and patients with schizophrenia. Cereb Cortex 2002;12:1218-24

35. Park HJ, Westin CF, Kubicki M, et al. White matter hemisphere asymmetries in healthy subjects and in schizophrenia: a diffusion tensor MRI study. $\mathrm{Neu}$ roimage 2004;23:213-23

36. Jones DK, Symms MR, Cercignani M, et al. The effect of filter size on VBM analyses of DT-MRI data. Neuroimage 2005;26:546-54

37. Kubicki M, Westin CF, Maier SE, et al. Uncinate fasciculus findings in schizophrenia: a magnetic resonance diffusion tensor imaging study. Am J Psychiatry 2002;159:813-20

38. Gong G, Jiang T, Zhu C, et al. Asymmetry analysis of cingulum based on scale-invariant parameterization by diffusion tensor imaging. Hum Brain Mapp 2005;24:92-98

39. Catani M, Howard RJ, Pajevic S, et al. Virtual in vivo interactive dissection of white matter fasciculi in the human brain. Neuroimage 2002;17:77-94

40. Kubicki M, Park H, Westin CF, et al. DTI and MTR abnormalities in schizophrenia: analysis of white matter integrity. Neuroimage 2005; 26:1109-18

41. Lu LH, Crosson B, Nadeau SE, et al. Category-specific naming deficits for objects and actions: semantic attribute and grammatical role hypotheses. Neuropsychologia 2002;40:1608-21 
42. Frith CD, Friston KJ, Herold S, et al. Regional brain activity in chronic schizophrenic patients during the performance of a verbal fluency task. Br J Psychiatry 1995; $167: 343-49$

43. Yurgelun-Todd DA, Waternaux CM, Cohen BM, et al. Functional magnetic resonance imaging of schizophrenic patients and comparison subjects during word production. Am J Psychiatry 1996;153:200-05

44. Ragland JD, Gur RC, Valdez J, et al. Event-related fMRI of frontotemporal activity during word encoding and recognition in schizophrenia. Am J Psychiatry 2004;161:1004-15

45. Romanski LM, Tian B, Fritz J, et al. Dual streams of auditory afferents target multiple domains in the primate prefrontal cortex. Nat Neurosci 1999;2: 1131-36

46. Catani M, Jones DK, ffytche DH. Perisylvian language networks of the human brain. Ann Neurol 2005;57:8-16

47. Duffau H, Gatignol P, Mandonnet E, et al. New insights into the anatomofunctional connectivity of the semantic system: a study using cortico-subcortical electrostimulations. Brain 2005;128:797-810

48. Tuch DS. Q-ball imaging. Magn Reson Med 2004;52:1358-72

49. Woods RP, Dodrill CB, Ojemann GA. Brain injury, handedness, and speech lateralization in a series of amobarbital studies. Ann Neurol 1988;23:510-18 